\title{
Review of Recent Advances in Vaginal Mesh Tissue Interaction
}

\author{
Arnab Chanda ${ }^{1 *}$ and Weston Upchurch ${ }^{2}$ \\ ${ }^{1}$ Department of Bio Engineering, USA \\ ${ }^{2}$ Department of Surgery, USA \\ *Corresponding author: Arnab Chanda, Department of Bio Engineering, Pittsburgh, PA-15213, USA
}

Submission: 眥February 9, 2018; Published: 眥 March 28, 2018

\begin{abstract}
Pelvic Organ Prolapse (POP) is a critical deformity of the female pelvic floor suffered by over millions of women in the US. POP leads to prolapse of pelvic organs onto the vaginal canal causing discomfort, pain, strain and sexual dysfunction. Vaginal meshes are traditionally implanted surgically to rectify the herniation of the pelvic organs and correct patient-specific POP conditions. However, the bio incompatibility of such meshes within the female pelvic system have been recognized to be deletarious to the surrounding tissues due to mesh erosion, organ perforation and tissue slicing, leading to severe complications. In literature, several studies have been conducted to understand vaginal mesh mechanical properties and mesh tissue interactions. The current article reviews these recent advances, which will not only be valuable to understand the state of the art in the mesh tissue interaction characterization and the directions in which further work needs to be conducted, but would also be indispensable for understanding the challenges associated with vaginal mesh failure and the corrective strategies in terms of design and implementation moving forward.
\end{abstract}

Keywords: Transvaginal mesh; Pelvic organ prolapse (POP); Female pelvic system

\section{Introduction}

A vaginal mesh is a net-like woven material which is surgically implanted through the vaginal opening to treat Pelvic Organ Prolapse (POP) condition. In POP, the female pelvic organs such as the urinary bladder, rectum, or the uterus prolapses onto the vaginal wall (Figure 1) causing vaginal discomfort, sexual dysfunction and strained urination and defaction [1]. The mesh creates a hammock- like structure under the drooping pelvic organs to fix them in place. However, traumatic mechanical failure of most such standard meshes within few months of implantation, accompanied with mesh induced complications such as organ perforation, tissue erosion and slicing, and prolapse relapse, have led to their discontinuation by the Food and Drug Administration (FDA) [2].

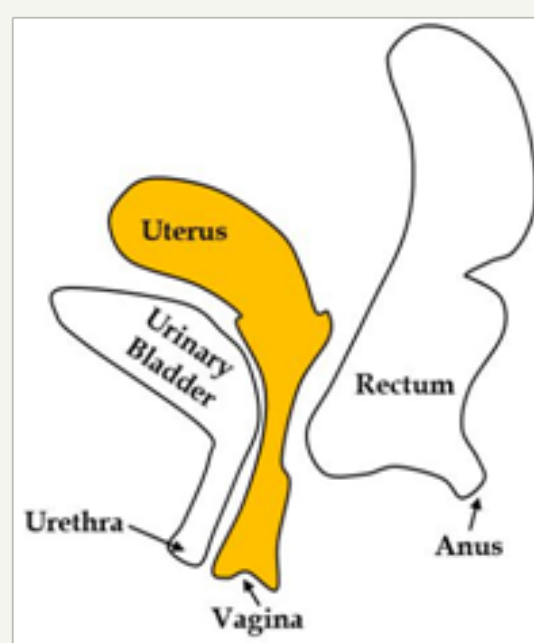

Normal Pelvic Anatomy

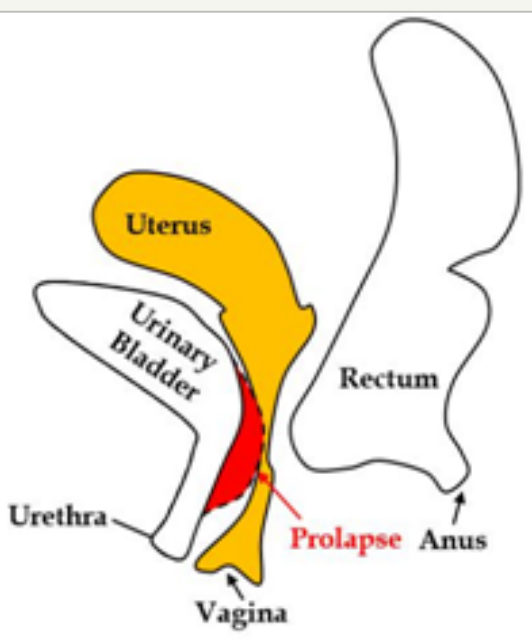

Anterior Vaginal Prolapse

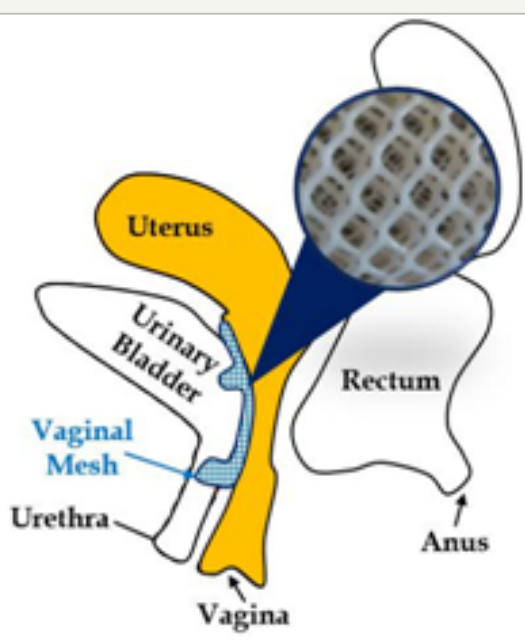

Prolapse Correction with Mesh

Figure 1: Schematic or normal pelvic anatomy, POP and mesh. 
Also, thousands of lawsuits have been filed against major mesh manufacturers namely Ethicon, Coloplast, Boston Scientific and American Medical Systems, not allowing patients to trust and avail vaginal meshes, and leading to major monetory losses. To improve the biofedelity of vaginal meshes, studies have been conducted to understand mesh mechanical properties, and its interaction with animal tissues. The upcoming sections will briefly cover these topics, and will be followed with conclusions and future directions of the vaginal mesh technology.

Mechanical testing and characterization of vaginal meshes is important for distinguishing the structural properties and material behaviour of the various mesh products available in the market. Extensive uni-axial, bi-axial and cyclic load testing's have been conducted on dry and wet meshes under different loads and boundary conditions. Rohrnbauer et al. [3,4] in 2014 studied the mechanical behaviour of prosthetic mesh at different length scales. A global mechanical testing framework was developed along with a methodology to estimate local deformation and kinematics. From the study, the global mechanical response of the mesh was found to depend on its anisotropic structure, non-linear force response, hysteresis and preconditioning effects. The local deformation analysis helped identify mesh mechanics at the unit cell level. Both the global and local kinematic responses of the mesh were observed to have direct relation with clinical observations such as mesh wrinkling, erosion and dislocation. Based on the study, various protocols were generated to characterize range of the mesh products available in the market.

Maurer etal. [3] in 2014 studied the mechanical biocompatibility of meshes using a robust experimental framework. It was found from the study that mesh porosity doesn't predict stiffness and mesh stiffness was observed to have a weak corellation with permanent deformation. Excessive biaxial stresses were found to lead to intense mesh shrinkage. Special emphasis was placed on carefully matching the mesh and underlying tissue stiffness to minimize discomfort. A very stiff mesh compared to the tissue was found to lead to stress shielding and shrinkage. On the other hand, a very compliant mesh was observed to lose their supportive function and affect mesh handling properties. To control the mechanical stiffness of an implanted mesh, embedding the mesh into a polymer matrix with preconditioning effect was suggested.

\section{Mechanical Tests on Meshes Explanted in Animal Models}

Mesh implantation was found by Morch et al. [5] to lead to the development of scar tissue and the formation of a new composite made of native tissue, the mesh implant and scar tissues. The influence of healing and healing time on the mechanical response under uniaxial tension of the new composite was studied in detail with various animal models [6-8]. Under small deformations, healing was found to have minimal influence on the composite mechanical properties. However, under large deformations, the composite was observed to stiffen at the last stage of healing (3-6 month span). It was concluded that the minimum time required to observe complete healing and a stable mechanical property for the composite was two months. In future, 3-6 month long mesh tissue interaction studies on animal models would suitable to appropriately understand biocompatibility of vaginal meshes.

\section{Conclusion and Future Directions}

In the current brief review, the criticality of understanding vaginal mesh tissue interaction ultimately aimed at improving mesh implantation outcomes for POP correction was discussed. The types of mechanical tests which have been conducted to date to understand mesh mechanical properties under varying loading conditions and at different scales were presented. One important observation mentioned based on the test results was the importance of improving the mesh material system by altering and matching its stiffness closer to the vaginal tissues.

Also, recent studies involving the mechanical interaction of meshes implanted into different animal models through various phases of healing was discussed. In future, further work needs to be conducted mainly in three areas to improve vaginal mesh biocompatibility. First, vaginal tissue surrogate materials [9-13] will have to be used along with additive manufacturing techniques to construct meshes [14]. Second, various bioactive and nano particle based coatings [15] have to be investigated for enhancement of mesh-tissue biocompatibility. Third, short and long term vaginal tissue erosion due to its mechanical interactions with meshes will have to be studied. Patient studies can follow only after such detailed investigations into the vaginal mesh technology.

\section{References}

1. Chanda A, Unnikrishnan V, Roy S, Richter HE (2015) Computational modeling of the female pelvic support structures and organs to understand the mechanism of pelvic organ prolapse: a review. Applied Mechanics Reviews 67(4): 040801.

2. Jacoby VL, Subak L, Waetjen LE (2016) The FDA and the vaginal mesh controversy-further impetus to change the $510(\mathrm{k})$ pathway for medical device approval. JAMA Intern Med 176(2): 277-278.

3. Maurer M, Röhrnbauer B, Feola A, Deprest J, Mazza E (2014) Mechanical biocompatibility of prosthetic meshes: A comprehensive protocol for mechanical characterization. J Mech behav biomed mater 40: 42-58.

4. Rohrnbauer B (2013) Mechanical characterization and modeling of prosthetic meshes. Technische Universität München, Germany.

5. Morch A, Pouseele B, Doucède G, Witz JF, Lesaffre F, et al. (2017) Experimental study of the mechanical behavior of an explanted mesh: The influence of healing. J Mech Behav Biomed Mater 65: 190-199.

6. Feola A, Abramowitch S, Jallah Z, Stein S, Barone W, et al. (2013) Deterioration in biomechanical properties of the vagina following implantation of a high-stiffness prolapse mesh. BJOG 120(2): 224-232.

7. Hernández Gascón B, Peña E, Pascual G, Rodríguez M, Bellón J, et al. (2012) Long-term anisotropic mechanical response of surgical meshes used to repair abdominal wall defects. J mech behav biomed mater 5(1): 257-271.

8. Deeken CR, Melman L, Jenkins ED, Greco SC, Frisella MM, et al. (2011) Histologic and biomechanical evaluation of crosslinked and non-crosslinked biologic meshes in a porcine model of ventral incisional hernia repair. J Am Coll Surg 212(5): 880-888.

9. Chanda A, Unnikrishnan V, Flynn Z (2016) Biofidelic skin stimulant. Google Patents. 
10. Chanda A, Flynn Z, Unnikrishnan V (2017) Biomechanical characterization of normal and prolapsed vaginal tissue surrogate. J Mech Med Biol DOI: doi.org/10.1142/S0219519417501007.

11. Chanda A, Callaway C, Clifton C, Unnikrishnan V (2016) Biofidelic human brain tissue surrogates. Taylor \& Francis DOI: doi.org/10.1080/153764 94.2016.1143749.

12. Chanda A, Unnikrishnan V (2016) Human tissue simulants for study of traumatic brain injury. (TBI) $31^{\text {st }}$ Technical Conference.

13. Chanda A, Unnikrishnan V, Flynn Z, Lackey K (2017) Experimental study on tissue phantoms to understand the effect of injury and suturing on human skin mechanical properties. Proc Inst Mech Eng H 231(1): 80-91.

14. Ballard DH, Weisman JA, Jammalamadaka U, Tappa K, Alexander JS, et al. (2016) Three-dimensional printing of bioactive hernia meshes: in vitro proof of principle. Surgery 161(6): 1479-1481.

15. Lo TS, Cortes EFM, Wu PY, Tan YL, Kharabsheh AA, et al. (2016) Assessment of collagen versus non collagen coated anterior vaginal mesh in pelvic reconstructive surgery: prospective study. Eur J Obstet Gynecol Reprod Biol 198: 138-144.
Creative Commons Attribution 4.0

International License

For possible submissions Click Here
Submit Article

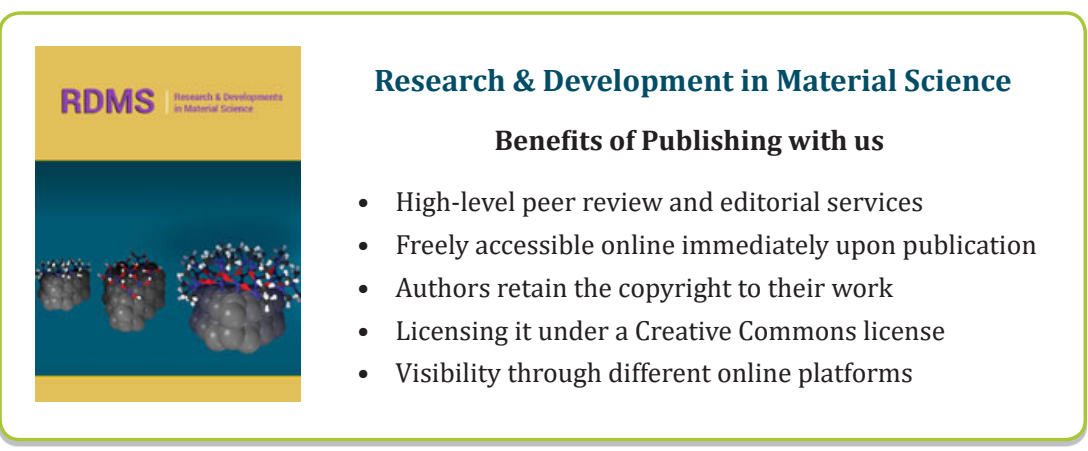

